
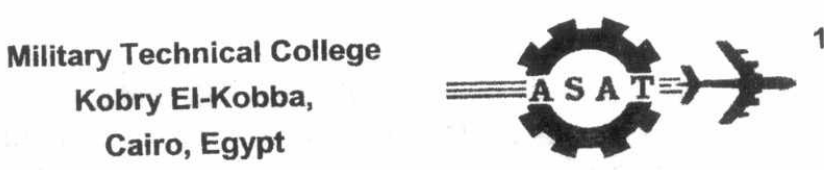

11-th International Conference

on Aerospace Sciences \&

Aviation Technology

\title{
DESIGN OPTIMIZATION OF COMPOSITE ROCKET MOTOR CASES USING GENETIC ALGORITHMS
}

\author{
El-Geuchy, M.I.* \\ Zayed, A-N.* \\ El-Maddah, M.M. ${ }^{\star *}$
}

\begin{abstract}
A new tool for designing a solid propellant rocket motor casing made of composite materials is obtained by merging the genetic algorithm optimization method with the finite element method. The genetic algorithm optimization method is used to determine the best design parameters of the laminate to minimize the weight of the rocket motor casing. The ANSYS finite element code is used to analyze the overstressing strength of the rocket motor casing. Tsai-Wu failure criterion is used to assess the first ply failure and an overstressing load level factor is introduced to describe the failure strength. Practical design examples, of composite rocket motor casings, are investigated using the present design tool.
\end{abstract}

KEYWORDS: Rocket motor case, Composite shell, Finite element, Genetic algorithm

* Egyptian armed forces

** Modern Academy in Maady 


\section{INTRODUCTION}

The design procedure of a solid propellant rocket motor which made of metal (steel, aluminum, and/or titanium) have been presented in [1]. In service, the rocket motor case (RMC) is commonly subjected to high internal pressure load, thermal load, and axial compressive thrust load during combustion. For spin stabilized rockets, the $\mathrm{RMC}$ is exposed to centrifugal forces too. It is also exposed to aerodynamic loads during flight.

Since the early seventies, composite materials have been used extensively to replace metals and reduce the inert weight of several aerospace and missile structures $[2,3]$. The design of missiles structures, made of composite materials, has been presented in $[4,5,6]$, and the failure analysis of the RMC is also presented in $[7,8,9]$.

Through the last thirty years, much works and processes had been made using the finite element method (FEM) $[10,11]$. Today, many finite element codes are used for general and specific purposes. Some of the general purpose codes are ABAQUS, SAP, ANSYS, MARC, and NASTRAN. In this study, ANSYS is chosen as a tool of analyzing the RMC made of composite materials.

This paper presents a merge of the finite element method (FEM) as an analyzing tool with a genetic algorithm $[12,13]$ as a design optimization tool. Genetic algorithms are subsets of evolutionary algorithms [14]. Genetic algorithms seek the optimal solution through random probability methods without auxiliary information such as derivatives or intelligently chosen starting points $[15,16]$.

\section{STRESS ANALYSIS OF A COMPOSITE ROCKET MOTOR CASE}

The laminated shell of the composite RMC is composed of a stack of filament wound layers with various orientations. The material coordinate system "1-2-3", as shown in Fig. 1, are: Axis "1" lies along the fiber direction and axes "2-3" are directed perpendicular to "1". It differs from layer to layer depending upon the orientation of the fibers, which is defined by the orientation angle " $\theta$ ".

In this study, a composite cylindrical RMC of length " $\mathrm{L}$ ", mean diameter " $\mathrm{D}$ " is considered and investigated, as shown in Fig. 2.

Let the mid-surface of the cylindrical shell of the RMC be the reference surface, and let the origin of the coordinates be located at one end of the cylinder. The laminate reference coordinates $\mathrm{x}, \mathrm{y}$, and $\mathrm{z}$ are chosen in the axial, hoop, and radial directions, respectively.

The static linear analysis of the composite RMC laminate is done using version 7 of ANSYS. It is a general purpose FE-program for static, dynamic as well as multiphysics analysis. ANSYS is used as an evaluation tool for any design proposed by the implemented optimization method.

In the finite element modeling, the RMC is modeled with a multi-layered shell element "shell-91". The element is defined by eight nodes as shown in Fig. 3. It has six degree of freedom at each node. The real constants of that element are specified where its real constants are the number of layers, the thickness, and the orientation 
angle of each layer. The used material properties are given. The model is meshed with a suitable mesh size and shape. Boundary conditions and loads are applied then the problem is solved. The elements, of middle zone of the solved model, are concerned to avoid ends effect.

The composite RMC laminate is subjected to internal pressure load, axial compressive thrust load, in addition to centrifugal forces due to spin effect[1].

- Due to internal pressure, a design pressure load " $\mathrm{P}$ " is acting on the inner surface of the FE-model, and an axial load per unit length " $\mathrm{N}_{\mathrm{a}}$ " acts in axial direction

$$
\mathrm{N}_{\mathrm{a}}=\frac{\mathrm{PD}}{4}
$$

- Due to thrust load, an axial load per unit length " $\mathrm{N}_{\mathrm{Ta}}$ " acts in axial direction

$$
\mathrm{N}_{\mathrm{Ta}}=\frac{-\mathrm{T}}{\pi \mathrm{D}}
$$

- Due to spinning effect, a hoop load per unit area " $\mathrm{N}_{\mathrm{hc}}$ " acts on the inner surface of the FE-model

$$
\mathrm{N}_{\mathrm{hc}}=2 \rho \pi^{2} \mathrm{Dn^{2 } \mathrm { t }}
$$

where $\mathrm{n}$ : the rotation per second around missile longitudinal axis

$\rho$ : the density of the composite material

To assess the capability of the fiber-reinforced composite cylindrical RMC to withstand failure due to overstressing, Tsai-Wu failure criteria $[9,17]$ is employed. A strength failure load factor " $\zeta$ " is introduced to identify the characteristics of the firstply failure of the cylindrical RMC. The first ply failure is considered the end of linear behavior of the laminate. Based on the Tsai-Wu failure criteria, the " $\zeta$ " is defined as,

$$
\zeta=\max _{k}\left[F_{11}\left(\sigma_{1}^{(k)}\right)^{2}+F_{22}\left(\sigma_{2}^{(k)}\right)^{2}+F_{66}\left(\sigma_{12}^{(k)}\right)^{2}+2 F_{12} \sigma_{1}^{(k)} \sigma_{2}^{(k)}+F_{1} \sigma_{1}^{(k)}+F_{2} \sigma_{2}^{(k)}\right]
$$

where $\sigma_{1}^{(k)}, \sigma_{2}^{(k)}, \sigma_{12}^{(k)}$ are the stresses components of the $k^{\text {th }}$ layer in the material coordinates. The strength parameters $F_{11}, F_{22}, F_{66}, F_{1}, F_{2}$, and $F_{12}$ are given as:

$$
\begin{aligned}
& F_{11}=\frac{1}{\sigma_{1 U} \sigma_{1 U}^{\prime}}, \quad F_{22}=\frac{1}{\sigma_{2 U} \sigma_{2 U}^{\prime}}, \quad F_{66}=\frac{1}{\sigma_{12 U}^{2}} \\
& F_{1}=\frac{1}{\sigma_{1 U}}-\frac{1}{\sigma_{1 U}^{\prime}}, \quad F_{2}=\frac{1}{\sigma_{2 U}}-\frac{1}{\sigma_{2 U}^{\prime}} \\
& F_{22}=-\frac{1}{2} \frac{1}{\sqrt{\sigma_{1 U} \times \sigma_{1 U}^{\prime} \times \sigma_{2 U} \times \sigma_{2 U}^{\prime}}}
\end{aligned}
$$

where $\sigma_{1 U}, \sigma_{1 U}^{\prime}, \sigma_{2 U}, \sigma_{2 U}^{\prime}$ are the tensile and compressive strength in the longitudinal and transverse directions and $\sigma_{12 U}$ is the in-plane shear strength. To insure the first ply failure does not occur in the RMC laminate, the following condition must be satisfied:

$$
\zeta \leq 1
$$


Equation (6) Is used as a constraint on the design optimization problem. The reciprocal of " $\zeta$ " is defined as the strength index of the RMC laminate. It gives indication about how far that laminate can withstand the applied loads.

The overstressing strength coefficient is calculated for each layer in every element in the concerned zone. The maximum overstressing strength coefficient " $\zeta$ " is sought and stored to be used by the optimization method.

A subroutine is written to perform the finite element analysis by ANSYS command language interface, called APDL language. APDL stands for ANSYS Parametric Design Language, a scripting language that is used to automate common tasks or even build a model in terms of parameters (variables). Building the model in terms of parameters permits the usage in design optimization codes easily.

\section{OPTIMIZATION METHODS}

Using optimization methods is more attractive since these methods can handle a large class of design problems. Design optimization methods are widely used in design of composite structures [13]. Optimization methods can be divided into two categories: global methods and local methods [12].

Global methods, such as those based on the genetic algorithm (GA), are aimed at obtaining the global optimum. These methods are most useful for problems in which multiple minima/maxima are present in the design space, where in real life the majority of design problems have multiple objectives.

Local methods require information on the gradient of the objective for locating the optimum which is the most obvious limitation for applying these methods. These gradient-based optimization must start with a specific parameterization of the design and the results depend on that starting point. For problems with multiple minima / maxima (multimodal objective functions), local methods are limited to produce only one of the objective (i.e., the local optimum), the actual value of which depends on the starting point of the optimization process. These methods therefore need several starting points so that to become trapped to local optimum.

\subsection{Genetic Algorithms}

Genetic algorithms have been established that they are more robust in their search than other conventional algorithms. Specially, in their search in multimodal, real valued search space. They depend on the principle of "survival of the fittest".

For optimization using GA, an initial population of design points (called individual or chromosomes) explores the search space for optimum solutions by means of a randomized process of selection, crossover, and mutation. The fitness values of each individual are calculated. Selection process selects individuals of superior fitness to reproduce more than others. Selected individuals generate new offspring by altering the genes (design parameters) on a chromosome. 


\section{Problem formulation}

The RMC, made of composite laminate is produced by filament winding of the laminate layers (plies) on a mandrel. The objective of this optimization problem is to find a composite RMC of minimum weight subjected to overstressing strength constraint due to the applied loads.

That design is specified according to the chosen design parameters of the laminate. The main design parameters discussed here are:

- The minimum number of layer required to withstand the applied loads.

- The orientation angles of each layer of the composite laminate that may vary form $\left(0^{\circ}: 90^{\circ}\right)$.

The maximum overstressing strength constraint " $\zeta$ " is calculated using the finite element subroutine, written by ANSYS APDL language. Generally, GAs are used to solve unconstrained optimization problems and to maximize a fitness function. It serves as an evaluation function in successive genetic operations.

In this study, a penalty function based on feasibility is used to transform the present constrained optimization problem into unconstrained one. The approach proposed by Deb [14], is used after appropriate modifications. These modifications are done to be convenient with the present optimization problem. Mathematically, the fitness function may be in the form as shown in Eq. (7).

where

$$
\text { Fitness }=\frac{q}{F(x)}=\left\{\begin{array}{cc}
\frac{q}{n-g(x)} & \text { if } g(x) \geq 0 \\
\frac{q}{(n+1)+r\left[g_{2}(x)\right]^{2}} & \text { otherwise }
\end{array}\right.
$$

$$
\begin{gathered}
g(x)=1-\zeta \\
g_{2}(x)=\left(1-\frac{1}{\zeta}\right)+\left|1-\frac{1}{\zeta}\right|
\end{gathered}
$$

$x=\{n, \theta\}$ is the design variable vector, $n$ is the total number of winding layers and $\theta$ is the orientation angle of helical winding layers. $F(x)$ is the modified objective function, $g(x)$ and $g_{2}(x)$ are the penalty functions, $q$ is a normalizing constant, $r$ is penalty parameter.

\subsection{Operation of the Genetic Algorithm}

There are different evolution strategies in GA [12]. These strategies try to mimic natural evolution of living beings. Steady-state GA is defined as an overlapping population type [15]. The amount of overlap between generations (iteration of the $\mathrm{GA}$ ) is specified by specifying the percentage of the population that should be replaced at each generation. In this report, steady-state GA is implemented with $50 \%$ overlap between generations. 
When using a GA, it must be able to represent a single solution to the problem in a single data structure (which represents the chromosome). The concept of Binary GA is to encode the genes into binary digits and the GA work with these coded genes. The Continuous Parameter GA differs in that the genes are represented by the actual used values without encoding. Both types are powerful and it is not difficult to find advocates for each [16,18].

In this paper, continuous parameter GA is adapted to be more convenient to represent the design parameters, which are a blend of continuous, represented in fiber orientation, and discrete parameters, represented in number of layers.

Crossover offspring by combining features of both chromosomes. One-point crossover combines two chromosomes by choosing one random cut-point; two-point crossover combines chromosomes by choosing two random cut-points; and uniform crossover combines chromosomes by choosing random cut-points, as presented in Fig. 4, uniform crossover is used with a strong crossover effect; the crossover rate is $60 \%$.

Mutation expands the search space by random changes of value of genes in the chromosome that alter the chromosome overall (exploring the design space). It is an insurance policy against premature loss of important notation. Flip mutation is used with small mutation rate of $4 \%$, as shown in Fig. 5 .

The convergence method determines when the genetic algorithm should stop evolving. In other words, after how many iterations (generations) the optimum design is obtained. The used method uses the convergence percent of the population as the stopping criterion. The flow chart for process of optimum design is shown in Fig. 6 . For the current work, the GA optimization method is implemented using an already existing C++ Library of GA called "GAlib version 2.45 by Wall 1996" [19].

\section{NUMERICAL EXAMPLES}

A practical design example of composite RMC laminate is discussed using the developed design optimization procedure. Then, the effects of different loading conditions on the optimum design are discussed. Finally, the obtained design tool is used to find the optimum design of a composite RMC laminate with isotensiod domes.

\subsection{A Practical Optimum Design Example of a Composite RMC Laminate}

The RMC studied in this example is made of only helical winding layers oriented at $\pm \theta$, and subjected to internal pressure. It is required to obtain the optimum value of $\theta$ and the number of layers using GA.

The FE model shape of the RMC is right angle cylindrical shell, as shown in Fig. 7. Due to model symmetry, half the length of the cylindrical part of the RMC is modeled. The model is of mean diameter $D=116 \mathrm{~mm}$, and length $L=345 \mathrm{~mm}$, which is half the length of the modeled RMC. The cylinder is fixed in axial direction at one end (due to symmetry condition) and free at the other end. A point on the fixed end has totally fixed degree of freedom to prevent insufficient fixation of the model. The applied internal design pressure $\mathrm{p}_{\mathrm{d}}$ of $15 \mathrm{MPa}$ acting on the RMC inner surface. 
An axial force "Na" corresponding to the applied pressure is applied on the free end to simulate the pressure vessel biaxial state of stresses.

The material properties used here are those of the unidirectional carbon/epoxy composite given in Table 1, obtained from [20]. The GA parameters are given in Table 2.

The method is converged after $\mathbf{4 3}$ generations and the optimum design point is:

- The orientation angles of layers $\theta= \pm 53^{\circ}$

- The number of layers $n=16 \quad$ where $\zeta=0.9571$

The charts of convergence to this design point are shown in Fig. 8. Figure 8(a) and (b) show that the initial proposed design parameters randomly cover their domains then during evolution process, the proposed parameters are gradually converged to the optimum values.

Table 1 Lamina properties for some advanced composites [20]

\begin{tabular}{|l|c|c|c|}
\hline \multicolumn{1}{|c|}{$\begin{array}{c}\text { The material } \\
\text { properties }\end{array}$} & $\begin{array}{c}\text { Glass/Epoxy } \\
\text { [S2-glass/P251S] }\end{array}$ & Kevlar/Epoxy & Carbon/Epoxy \\
\hline$E_{1}[\mathrm{GPa}]$ & 51 & 72 & 132.5 \\
\hline $\mathrm{E}_{2}[\mathrm{GPa}]$ & 17 & 5 & 10.8 \\
\hline $\mathrm{G}_{12}[\mathrm{GPa}]$ & 7 & 2 & 5.7 \\
\hline$v_{12}$ & 0.25 & 0.41 & 0.24 \\
\hline$\sigma_{1 \mathrm{U}}[\mathrm{MPa}]$ & 1779 & 1151 & 1515 \\
\hline$\sigma_{1 U}^{\prime}[\mathrm{MPa}]$ & -641 & -281 & -1010 \\
\hline$\sigma_{2 U}[\mathrm{MPa}]$ & 58 & 12 & 43.8 \\
\hline$\sigma_{2 U}^{\prime}[\mathrm{MPa}]$ & -186 & -134 & -87.6 \\
\hline$\sigma_{12 U}[\mathrm{MPa}]$ & 75 & 443 & 86.9 \\
\hline Density $\left[\mathrm{kg} / \mathrm{m}^{3}\right]$ & 1980 & 1350 & 1530 \\
\hline Fiber volume $[\%]$ & 60 & 60 & 60 \\
\hline
\end{tabular}

${ }^{*}$ Assuming the same layer thickness for all fiber types $=0.13 \mathrm{~mm}$.

\section{Effect of orientation angle on optimum design of a RMC}

It is required to see how the orientation angle design parameter affects the strength of the RMC laminate. This effect could be identified by varying the orientation angle from $0^{\circ}$ : $90^{\circ}$ with incremental increase and analyzing every design point using ANSYS. Figure 9 shows the variation of the strength index, which is the inverse of " $\zeta$ ", with the orientation angle for the obtained optimum RMC of 16 layers. The variation is identical to results obtained by [7] which verify the used FEM. The maximum strength index is at the optimum orientation angle found by the GA optimization method.

It is also required to compare the optimum orientation angles when using different types of composites stated in Table 1. The results are stated in Table 3 . The values of the optimum orientation angles differ from each other and differ from the optimum 
angle stated by the netting theory. It stated that the value of optimum winding angle $\theta=54.74^{\circ}$ when the internal pressure is only the applied load [4]. This differences because netting theory neglects the effect of the matrix material. The effect of resinfiber interaction yields for that variation.

It is also found that using carbon/epoxy composite material yields to minimum RMC weight. Figure 9 demonstrates the strength index variation with orientation angle and the optimum orientation angles for the used composites.

Table 2 GA parameters

\begin{tabular}{|l|c|}
\hline Data structure of chromosome & $\begin{array}{c}\text { Continuous parameter GA } \\
\text { Evolution strategy }\end{array}$ \\
\hline Selection strategy & $\begin{array}{c}\text { Steady-state GA } \\
\text { with } 50 \% \text { overlap between generations }\end{array}$ \\
\hline Crossover strategy & Roulette wheel \\
\hline Mutation strategy & Uniform crossover with crossover rate $60 \%$ \\
\hline Population size & Flip mutation with mutation rate of $4 \%$ \\
\hline Convergence method & 20 \\
\hline Length of chromosome & Convergence percent of the population \\
\hline Range for gene 1 (orientation angle) & 2 genes \\
\hline Range of gene 2 (number of layers) & $5^{\circ}: 85^{\circ}$ \\
\hline
\end{tabular}

Table 3 Optimum configurations of the laminates for different types of composite materials

\begin{tabular}{|c|c|c|c|c|}
\hline $\begin{array}{c}\text { Composite } \\
\text { material }\end{array}$ & $\begin{array}{c}\text { Optimum } \\
\text { angle [Deg] }\end{array}$ & $\begin{array}{c}\text { No. of } \\
\text { layers }\end{array}$ & $\begin{array}{c}\text { Wall } \\
\text { thickness[mm] }\end{array}$ & $\begin{array}{c}\text { Mass } \\
\text { [kg/m] }\end{array}$ \\
\hline S-Glass / Epoxy & 58.2 & 34 & 4.42 & 3.189 \\
\hline Kevlar / Epoxy & 52 & 30 & 3.9 & 1.919 \\
\hline Carbon / Epoxy & 53 & 16 & 2.08 & 1.16 \\
\hline
\end{tabular}

\subsection{Effect of Applied Loads on Laminate Optimum Design}

An introduced axial compressive thrust force or spinning of RMC with specified angular speed will affect the optimum laminate configuration. The effect of the axial thrust force and angular rotation on strength index of a composite laminate of 16 layers is shown in Fig. 10 and Fig. 11.

By increasing the value of the axial compressive thrust force, the strength index increases and the optimum angle is shifted towards the hoop-winding angle. This is because the compressive load opposes the generated axial force due to internal pressure. The affected region is for the orientation angle more than $50^{\circ}$.

For angular rotation, there is a small effect on the optimum design. This is because composite materials have lower densities, and density is the main effective 
parameter for the generated centrifugal load per unit area, $\mathrm{N}_{\mathrm{hc}}$, stated in Eq. 3 . The strength index is generally decreased and the optimum angle is shifted towards the hoop winding angle when the angular rotation speed is increased.

\subsection{Design Optimization of Composite RMC Laminate with an Isotensoid Dome}

The RMC studied here having the cylindrical part with partially or totally closed ends, called "domes", are made of a composite material as shown in Fig. 12. It is made of helical and polar windings. The helical winding layers are oriented at predefined $\pm \theta$. To have an isotensoid dome, the helical winding angles are specified using the following equation [4].

$$
\theta=\sin ^{-1} \frac{R_{p}+B W / 2}{D / 2}
$$

where $R_{p}$ is the radius of the polar opening of the dome, and BW is the width of the fiber band used in filament winding process.

The RMC geometry, loads and the used materials are the same as those of the practical design example. The design parameters in this example are:

- The optimum number of hoop winding layers, $N_{\text {hoop }}$

- The optimum number of helical winding layers, $N_{\text {helix }}$

The objective is to find the optimum values for these two parameters for every predefined value of $\theta$ using GA. The proposed design tool is modified to solve the assigned problem and the results are as shown in Table 3.

Table 3 The laminate total number of layers, the optimum number of helical and hoop layers for every assigned orientation

\begin{tabular}{|c|c|c|c|c|c|c|}
\hline $\begin{array}{c}\text { Orientation } \\
\text { angle } \theta^{\prime \prime}\end{array}$ & $\begin{array}{c}\text { No. of } \\
\text { helical } \\
\text { layers }\end{array}$ & $\begin{array}{c}\text { No. of } \\
\text { hoop } \\
\text { layers }\end{array}$ & $\begin{array}{c}\text { Total } \\
\text { no. of } \\
\text { layers }\end{array}$ & $\zeta^{\prime 2}$ & Eitness & BW W Formm \\
\hline 0 & 5 & 14 & 19 & 0.992 & 5265.49 & 0 \\
\hline 15 & 6 & 13 & 19 & 0.912 & 4985.76 & 12.51 \\
\hline 30 & 11 & 10 & 21 & 0.986 & 4764.99 & 26.50 \\
\hline 45 & 12 & 7 & 19 & 0.954 & 5276.02 & 38.51 \\
\hline 53 & 16 & 0 & 16 & 0.957 & 6266.80 & 43.82 \\
\hline 60 & 25 & 0 & 25 & 0.996 & 4000.68 & 47.73 \\
\hline 75 & 56 & 0 & 56 & 0.963 & 1712.40 & 53.52 \\
\hline 85 & 70 & 0 & 70 & 0.978 & 746.39 & 55.28 \\
\hline
\end{tabular}

Figure 13 shows the laminate total number of layers, the optimum number of helical and hoop layers for every assigned winding angle. It is shown that the number of total layers of the composite laminate increased highly if the required winding angle is higher than $53^{\circ}$, the optimum angle for carbon/epoxy composite laminate. This is because the helical winding layers are responsible for carrying the axial loads, so 
going far from that optimum winding angle, the ability of helical winding layers to carry axial loads decreases, leading to require more layers to carry that load.

So, it is preferred not to use winding angle more than the optimum one. This means that the polar radius of the RMC and the used fiber bandwidth should be chosen so that the value of the winding angle does not exceed the optimum one.

\section{CONCLUSIONS}

- The merge, of the finite element method with a genetic algorithm, gives a good tool for analyzing and optimizing any design of a solid propellant rocket motor made of composite materials.

- The continuous parameter GA is considered as an efficient search technique for getting more accurate optimum solutions. The laminate main configurations for a composite RMC are easily obtained without auxiliary information such as derivatives of the objective function or an initial guessing point.

- The fiber orientation design parameter highly affects the strength of the RMC. In addition, it is found that the obtained design tool gives more accurate results than netting theory, which stated that the optimum value of $\theta=54.75^{\circ}$ for internal pressure load. The reason for that difference in results is because the netting theory neglects the effect of the used resin matrix material.

\section{REFERENCES}

[1] Zayed, A-N., El-Maddah, M.M. and El-Geuchy, M.I., "Design Procedure For Solid Propellant Combustion Chamber", $10^{\text {th }}$ Int. Conf. on Aerospace Sciences \& Aviation Technology, MTC, Cairo, Egypt, 2003.

[2] Sutton, G.P., "Rocket Propulsion Elements", 6-th Edition, John Wiley \& Sons, ISBN 0-471-52938-9, 1992.

[3] Policelli, F.J. and Vicario, A.A., "Applications of Composite Materials in Space And Missile Systems", Technical Report, Hercules Aerospace Company, 1987.

[4] Evans, P.R., "Composite Motor Case Design", AGARD-Lecture-Series, No 150, 1987.

[5] Sun, X.K., Du, S.Y. and Wang, G.D., "Bursting Problem of Filament Wound Composite Pressure Vessels", Int. J. of Pressure Vessels \& Piping, pp 55-59, 1999.

[6] Nyssen, C., "Non Linear Incremental Analysis Up to Failure of Aeronautical Structures", Computers \& Structures, Vol. 12, pp 593-605, 1980.

[7] Parnas, L. and Katirci, N., "Design of Fiber-Reinforced Composite Pressure Vessel under Various Loading Conditions", Composite Structures, Vol. 58, pp 83-95, 2002.

[8] Swanson, S.R., "Strength Design Criteria for Carbon/Epoxy Pressure Vessels", J. Spacecraft \& Rockets, Vol. 27(5), pp 522-526, 1990.

[9] Jones, R.M., "Mechanics of Composite Materials", New York: McGraw-Hill; 1975. 
[10] Logan, D.L., "A First Course in the Finite Element Method", PWS-KENT Publishing Co., Boston, USA, ISBN 0-534-92964-8, 1992.

[11] Moaveni, S., "Finite Element Analysis", Prentice Hall, ISBN 0-13-785298-0, 1999.

[12] Goldberg, D.E., "Genetic Algorithms in Search, Optimization and Machine Learning", Addison-Wesley, 1989.

[13] Park, J.H., Hwang, J.H., Lee, C.S., and Hwang, W., "Stacking Sequence Design Of Composite Laminates For Maximum Strength Using Genetic Algorithms", Composite Structures, Vol. 52(2), pp 217-231, 2001.

[14] Deb, K., "An efficient constraint Handling Method for Genetic Algorithms", Computer Methods in Applied Mechanics and Engineering, Vol. 186, pp 311$338,2000$.

[15] DeJong, K.A., "An Analysis of the Behavior of a Class of Genetic Adaptive Systems", Ph.D. thesis, University of Michigan, 1975.

[16] Haupt, R.L., and Haupt, S.E., "Practical Genetic Algorithms", John Wiley \& Sons, ISBN 0-471-18873-5, 1998.

[17] Cho-Chung Liang and Hung-Wen Chen, "Optimum Design of Fiber-Reinforced Composite Cylindrical Skirts for Solid Rocket Cases Subjected to Buckling and Overstressing Constraints", Composites Part B: Engineering, Vol. 34 (3), pp 273-284, April 2003.

[18] Wahba, W. A., "Design Optimization of Centrifugal Pump Impellers using Parallel Genetic Algorithm", Ph.D. Thesis, Cranfield University, 2001.

[19] Wall, M., "GAlib: A C++ Library of Genetic Algorithm Components", version 2.4, Massachusetts Institute of Technology, USA, 1996.

[20] Peters, S. T., "Handbook of Composites", $2^{\text {nd }}$ Edition, Chapman \& Hall, ISBN 0412-54020-7, 1998. 

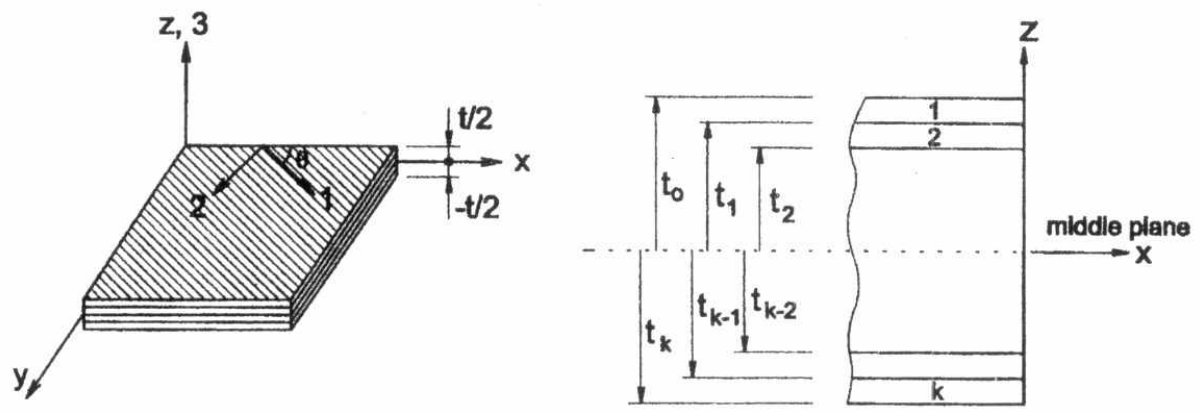

Fig. 1 Principal material axes with references axis and the stacking sequence

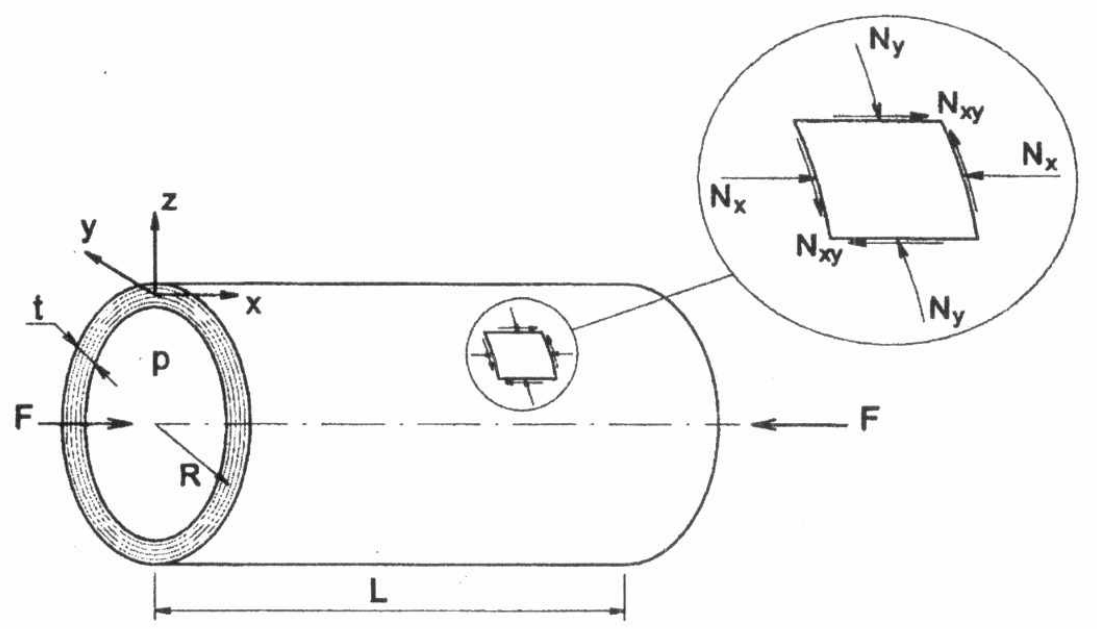

Fig. 2 The resultant forces of a circular cylindrical Rocket motor casing
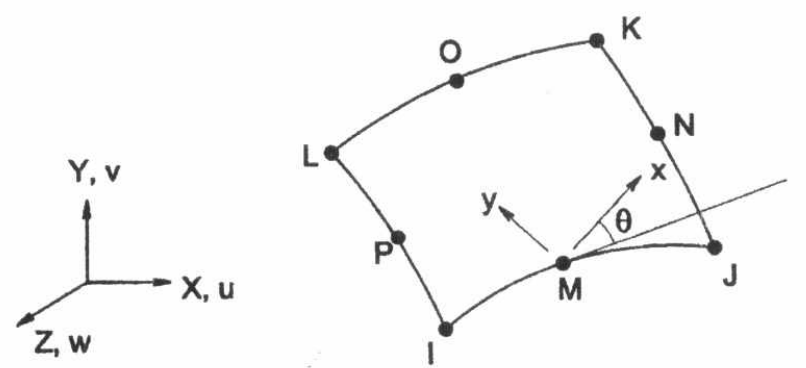

Fig. 3 Eight nodes, curved boundaries shell element "shell91" 


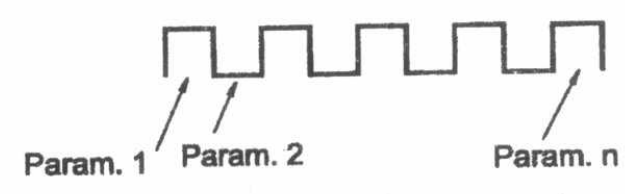

A chromosome of $n$ parameters
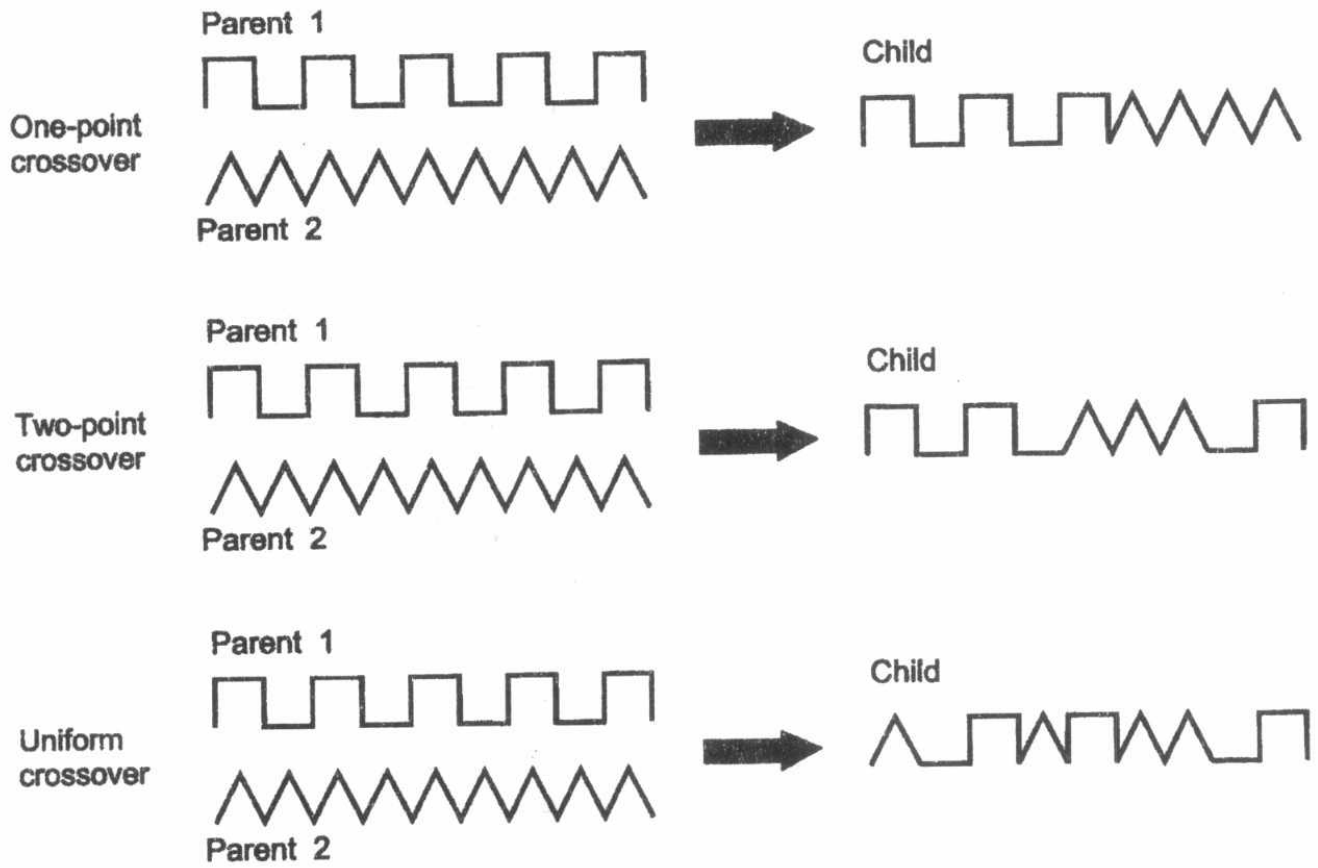

Fig. 4 Different crossover processes

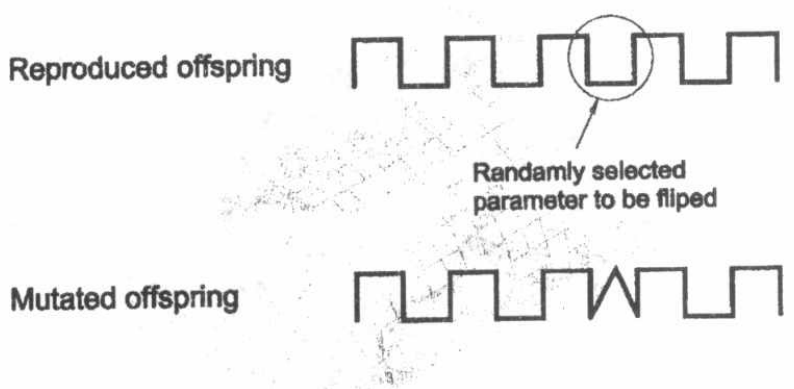

Fig. 5 Flip mutation process 


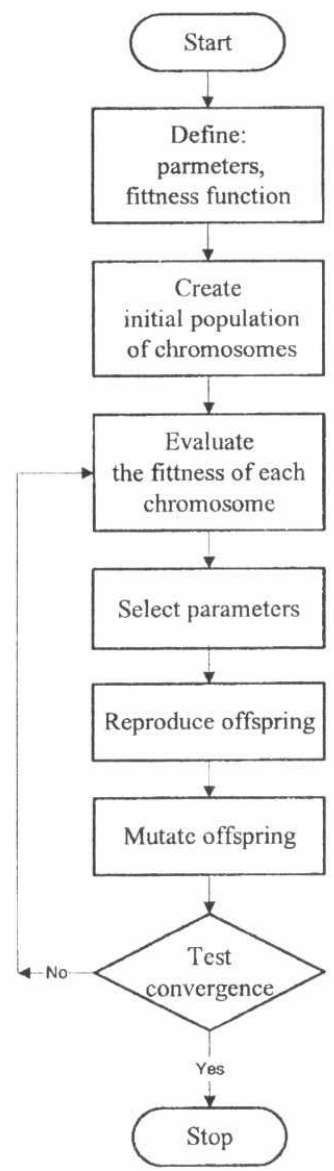

Fig. 6 Flowchart of a continuous parameter genetic algorithm

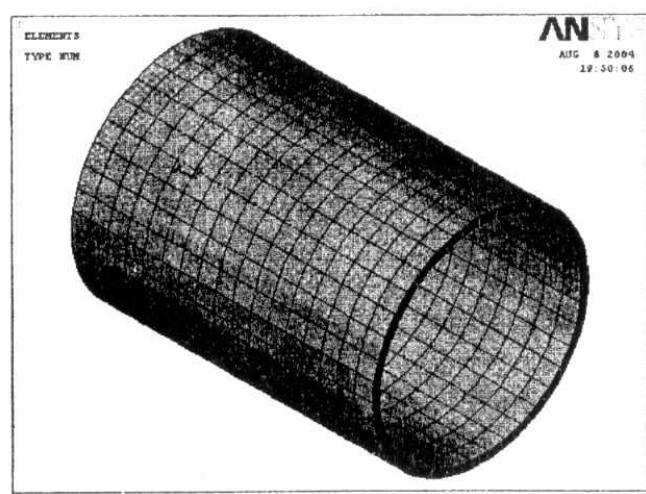

Fig. 7 The finite element model of the composite RMC 


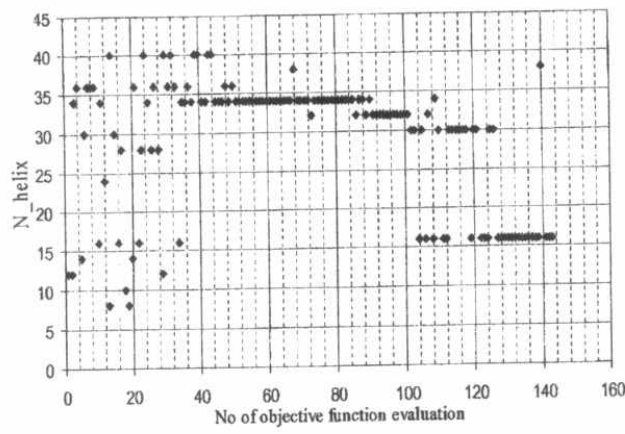

(a)

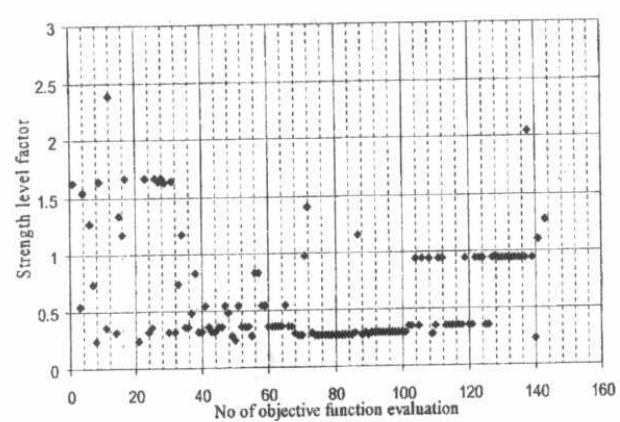

(c)



(b)

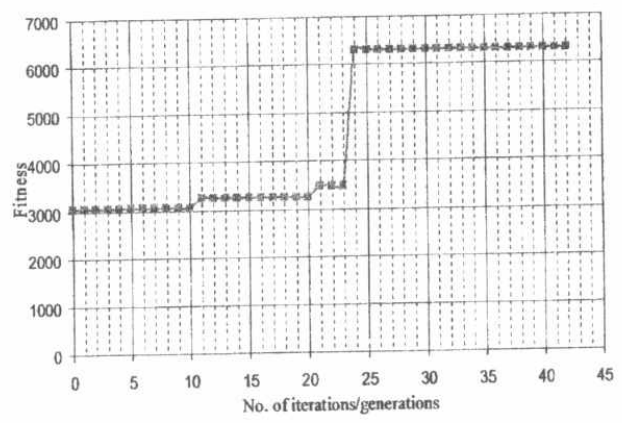

(d)

Fig. 8 Charts of convergence for GA optimization method, (a) variation of no. of layers, (b) variation of $\theta$, (c) variation of $\zeta$, (d) evolution of fitness value 




Fig. 9 Variation of the strength index with the orientation angle

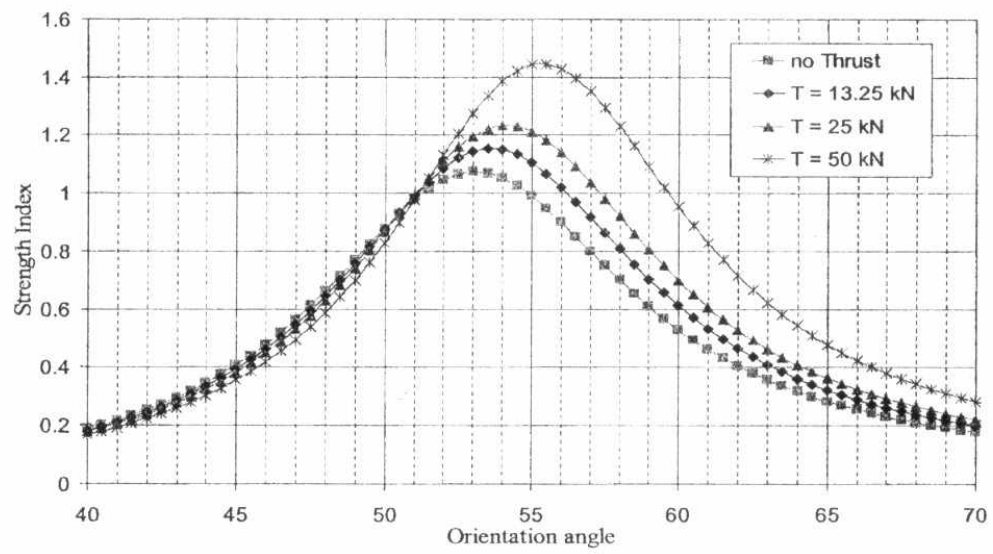

Fig. 10 Effect of axial thrust force on laminate optimum design

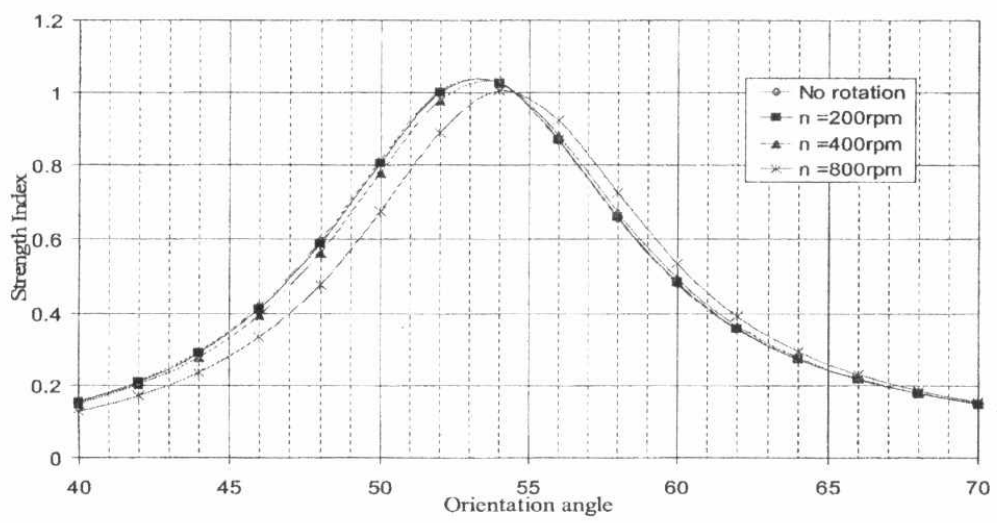

Fig. 11 Effect of angular rotation on laminate optimum design 
$1 / 2$ B.W

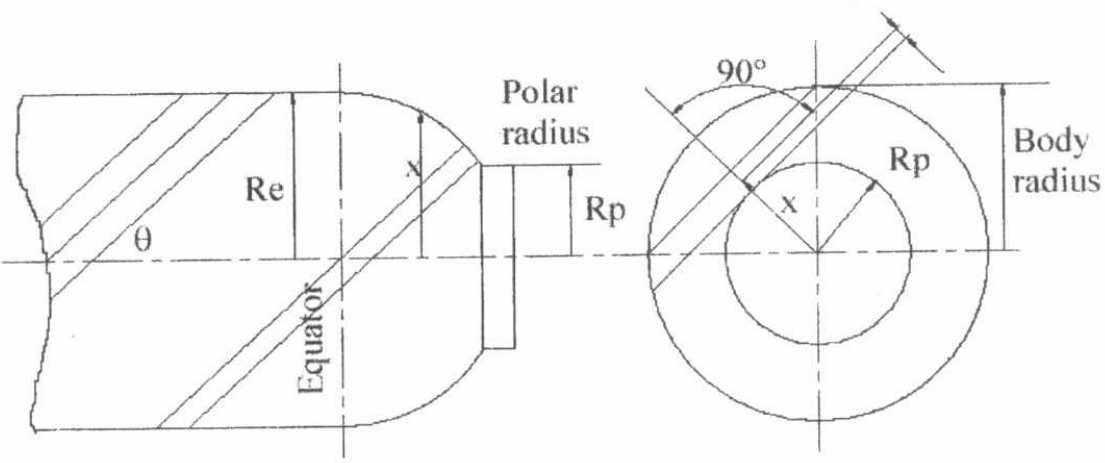

Fig. 12 The relative diameter of polar opening and case body determine the helical winding angle [4]

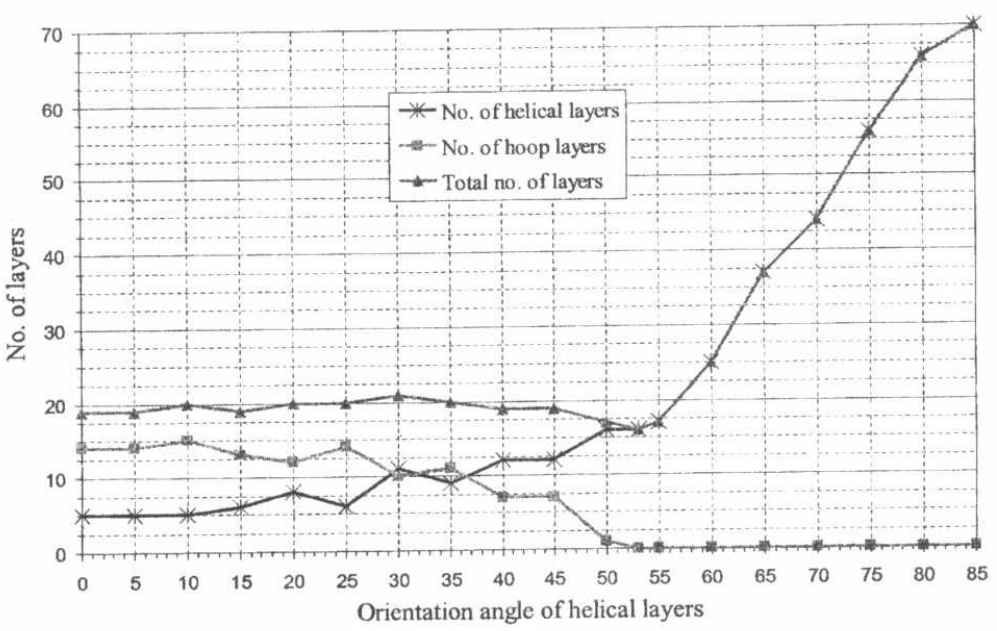

Fig. 13 Laminate optimum configuration for every assigned winding angle 